\title{
Admission clinicopathological data, length of stay, cost and mortality in an equine neonatal intensive care unit
}

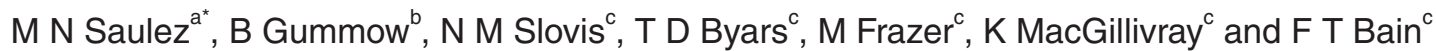

\begin{abstract}
Veterinary internists need to prognosticate patients quickly and accurately in a neonatal intensive care unit (NICU). This may depend on laboratory data collected on admission, the cost of hospitalisation, length of stay (LOS) and mortality rate experienced in the NICU. Therefore, we conducted a retrospective study of 62 equine neonates admitted to a NICU of a private equine referral hospital to determine the prognostic value of venous clinicopathological data collected on admission before therapy, the cost of hospitalisation, LOS and mortality rate. The WBC count, total $\mathrm{CO}_{2}\left(\mathrm{TCO}_{2}\right)$ and alkaline phosphatase (ALP) were significantly higher $(P \leq 0.05)$ and anion gap lower in survivors compared with nonsurvivors. A logistic regression model that included WBC count, hematocrit, albumin/ globulin ratio, $\mathrm{ALP}, \mathrm{TCO}_{2}$, potassium, sodium and lactate, was able to correctly predict mortality in $84 \%$ of cases. Only anion gap proved to be an independent predictor of neonatal mortality in this study. In the study population, the overall mortality rate was $34 \%$ with greatest mortality rates reported in the first 48 hours and again on day 6 of hospitalisation. Amongst the various clinical diagnoses, mortality was highest in foals after forced extraction during correction of dystocia. Median cost per day was higher for nonsurvivors while total cost was higher in survivors.
\end{abstract}

Key words: clinicopathology, critical care, equine, financial expense, intensive care unit, neonate, survival.

Saulez M N, Gummow B, Slovis N M, Byars T D, Frazer M, MacGillivray K, Bain F T Admission clinicopathological data, length of stay, cost and mortality in an equine neonatal intensive care unit. Journal of the South African Veterinary Association (2007) 78(3): 153-157 (En.). Department of Companion Animal Clinical Studies, Faculty of Veterinary Science, Onderstepoort, University of Pretoria, Private Bag X01, Onderstepoort, 0110 South Africa.

\section{INTRODUCTION}

In a Neonatal Intensive Care Unit (NICU), internists are under pressure to prognosticate patients accurately and timely. Initially, their decision to treat may depend on case history, physical examination and clinicopathological data. Laboratory data obtained on admission may reflect current pathophysiological status and can be useful in estimating risk of death $^{4,14,17,18}$.

In the veterinary setting, hospitalisation of a critically-ill foal at a university referral hospital is expensive with daily costs in the NICU ranging from 250 to more than US $\$ 1000^{8}$. The cumulative costs of increased metabolic monitoring, equipment, personnel and pharmaceutical agents may rapidly escalate costs, making repeated clinicopathological measurements either not possible or not frequently determined. Knowing how the initial clinicopathological data correlate

aDepartment of Companion Animal Clinical Studies and ${ }^{b}$ Section of Epidemiology, Faculty of Veterinary Science, Onderstepoort, University of Pretoria, 0110 South Africa. 'Hagyard Equine Medical Institute, Lexington, KY, USA.

${ }^{*}$ Author for correspondence.

E-mail: montague.saulez@up.ac.za

Received: June 2007. Accepted: August 2007. with the survival of a patient, the length of stay (LOS) and cost of hospitalisation, may guide the veterinary internist in the decision-making process.

Although previous studies have evaluated the prognostic value of clinicopathological data determined after admission in foals $<10$ days of age in a NICU ${ }^{11}$, foals with septicaemia ${ }^{8}$ and foals with pulmonary disease ${ }^{2}$, have determined multivariable models to assess survivability of neonatal foals admitted to a NICU $\leq$ 7 days $^{18}$ and $<14$ days of age $e^{7}$, and determined risk factors associated with the prognosis for survival and athletic use in foals with septic arthritis ${ }^{20}$, reports on the relationship between admission clinicopathological data, LOS and cost are lacking. The purpose of this study was to firstly investigate whether clinicopathological data collected on admission in untreated foals (age $<3$ days) could predict mortality and secondly to determine the average LOS and cost at a private referral equine NICU.

\section{MATERIALS AND METHODS}

Neonatal foals age $<72$ hours admitted to a NICU from January to July 2004 were studied. Venous clinicopathological data was determined before initiation of treatment, LOS (days), cost (US\$), final diagnosis, presence of septicaemia, and hospital outcome (alive or dead) were extracted from medical records. Clinicopathological data collected included haematology: WBC count, haematocrit, and platelet count (Abbott CELL-DYN ${ }^{\circledR}$ 3500, Abbott Diagnostics, USA); serum biochemistry: sodium, potassium, chloride, calcium and phosphate concentrations, total $\mathrm{CO}_{2}$ concentration $\left(\mathrm{TCO}_{2}\right)$, BUN concentration, creatinine concentration, glucose concentration, fibrinogen concentration, total plasma protein concentration, total protein concentration, gamma-glutamyltransferase concentration (GGT), sorbitol dehydrogenase concentration, alkaline phosphatase concentration (ALP), aspartate amino-transaminase concentration, lactate dehydrogenase concentration, creatine phosphokinase concentration $(\mathrm{CK})$, total bilirubin concentration, ammonia concentration, anion gap (calculated), albumin concentration, albumin/globulin ratio (A/G ratio) and immunoglobulin concentration (Olympus Au400e chemistry-immuno analyser, Olympus America Inc., USA); and whole blood lactate concentration (Accutrend ${ }^{\circledR}$ Lactate analyser, Roche Laboratories, USA). Survival was defined as those foals that were discharged alive from the NICU. Sepsis was defined as a positive admission blood culture $\left(\mathrm{BBL}^{\mathrm{TM}}\right.$ Septi-chek ${ }^{\mathrm{TM}}$ TSB, Becton Dickson Microbiology Systems, USA) or microbiological evidence of localised infection. Foals that were initially treated but showed clinical deterioration and prompted euthanasia as deemed necessary by the primary clinician were included in this study.

Data were stored in Microsoft Excel 2003 and analysed using Microsoft Excel and NCSS 2004. The survival rate of foals admitted to the NICU was assessed using the nonparametric Kaplan-Meier and Nelson-Aalen estimates of survival and associated hazard rates ${ }^{13}$. Descriptive statistics were performed on each of the blood parameters measured and data were tested for normality using the

${ }^{a}$ Hintze J. NCSS and PASS number cruncher statistical systems, Kaysville, Utah. http;//www.ncss.com, 2001. 
Shapiro-Wilk ${ }^{19}$ and the Anderson Darling $^{1}$ tests. Since many of the parameter data were not normally distributed, data were analysed as nonparametric data. Median scores between the survivor and nonsurvivor groups were therefore compared using the Wilcoxon Rank-Sum Test ${ }^{13}$ for difference in medians.

Logistic regression using (Hierarchical) Forward Switching was performed to access the correlation between parameters and mortality. Forward Switching is similar to the method of Forward Selection; however, at each step when a term is added, all terms in the model are switched one at a time with all candidate terms not in the model to determine if they increase the value of the log likelihood. If a switch can be found, it is made and the pool of terms is again searched to determine if another switch can be made. This switching was limited to those keeping the model hierarchical. When the search for possible switches does not yield a candidate, the subset size is increased by 1 and a new search is begun. The algorithm is terminated when a target subset size is reached or all terms are included in the model. The regression model included 17 parameters to begin with: lactate | WBC count | Ht | sodium | potassium | chloride $\left|\mathrm{TCO}_{2}\right|$ anion gap | glucose | BUN | creatinine | calcium | albumin | A/G ratio | ALP | GGT | CK |. A main-effects only model was analysed and no cross-products or interaction terms were included. The reference value for the model was survivors. The model was run several times varying the cut-off number of parameters (terms) included in the model. The model that produced the highest percentage of correctly classified cases (survived or died) was taken as the best model for predicting death. Because collinearity, or multicollinearity (near-linear relationships), was likely to exist between parameters, the data was also examined for these relationships using a Spearman Rank correlation matrix. Parameters that were found to be significant in the logistic regression model were then tested as individual predictors of mortality using empirical (nonparametric) receiver operator curves (ROCs) ${ }^{5}$. The area under a ROC curve (AUROC) is a popular measure of the accuracy of a diagnostic test. The larger the AUROC, the better the test is at predicting the existence of the disease. The possible values of AUROC range from 0.5 (no diagnostic ability) to 1.0 (perfect diagnostic ability). The confidence interval for these curves is based on the transformed AUROC as given by Zhou et al. ${ }^{22}$. AUROCs were compared to the value 0.5 to see if there was a statistically significant difference between the

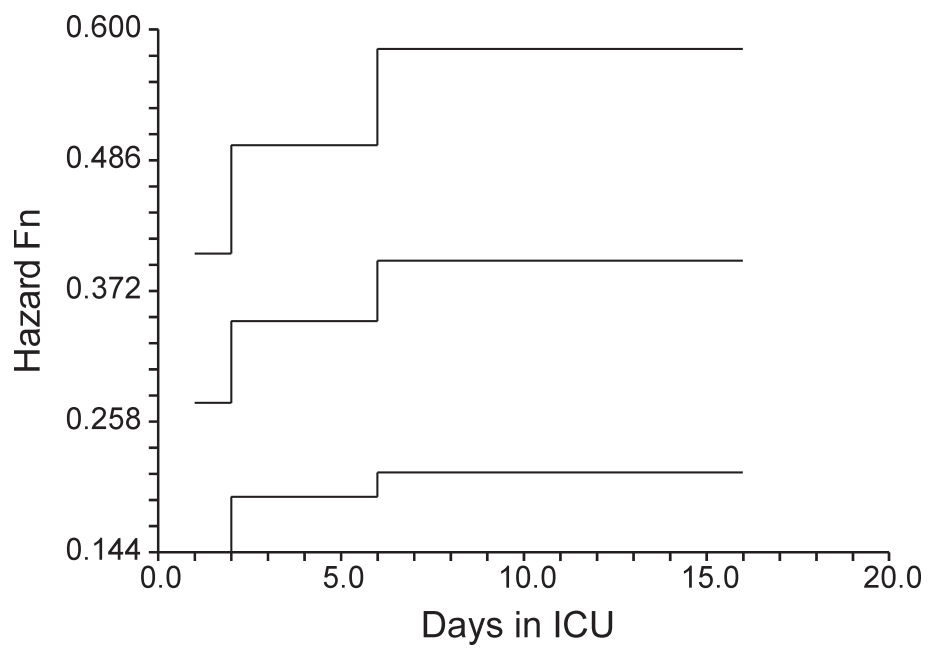

Fig. 1: Hazard function plot showing rate of death for foals admitted to the NICU as well as the point-wise $95 \%$ confidence intervals.

calculated AUROC and no diagnostic ability ${ }^{5}$.

Since the financial data failed to meet all the assumptions of normality, median values were compared using the MannWhitney U/Wilcoxon Rank Sum Test ${ }^{10}$. In addition, each group was classified into septicaemic and nonsepticaemic patients; and the difference in cost between septicaemic and nonsepticaemic patients was compared.

\section{RESULTS}

Sixty-two foals with various clinical diagnoses were included in this study. These included hypoxic ischemia encephalopathy $(n=14)$; prematurity $(n=4)$; weakness following delivery of the foal by laparohysterotomy $(n=6)$ and forced extraction $(n=11)$; weakness following delivery from mares with premature placental separation $(n=9)$ and placentitis $(n=3)$; and colic of unknown aetiology $(n=2)$. The remaining 13 foals had immune mediated thrombocytopenia, flexural deformity, bladder rupture, localised bacterial infection (aspiration pneumonia), angular limb deformity, meconium impaction, failure to suckle, myopathy, acute respiratory distress syndrome, Tyzzer's disease, acute renal failure, bacteraemia and failure of passive transfer. Eight foals were concurrently affected by bacteraemia. Forty-one foals $(66 \%)$ survived to discharge while 21 foals (34\%) died.

Figure 1 shows the results of the Kaplan-Meir rates of death for foals admitted to the NICU. There was a $27 \%$ (95\% CI: $14-40 \%$ ) probability of a foal dying by the end of the first day and a $35 \%$ (95 \% CI: 19-50 \%) chance of them dying by the end of the second day after admission to the NICU. If they survived the first 2 days then their chance of dying did not increase until day 6 , when further mortalities are predicted. The chance of a foal dying by the end of day 6 after admission was $40 \%$ (95\% CI: 21 to $58 \%$ ). There was very little chance of foals dying after day 6. The hazard function plot (Fig. 1) showed that the critical care periods are therefore the first 48 hours and day 6 of admission to the NICU.

Table 1 compares the blood parameters of foals that survived with those that died using the Wilcoxon rank sum test. The WBC count, $\mathrm{TCO}_{2}$, and ALP were significantly higher in foals that survived $(P \leq$ $0.05)$ while the anion gap was significantly higher in foals that died $(P<0.05)$. No difference could be shown between the medians of the other blood parameters.

Table 2 shows the results of the logistic regression after forward switching to remove parameters that are poorly associated with predicting mortality. Using this process of elimination it was found that a model with the subset of 8 parameters as shown in Table 2 produced the highest percentage $(84 \%)$ of correctly classified cases $(88 \%$ of survivors and $76 \%$ of mortalities were correctly classified). The model's predictive ability is further illustrated by the high AUROC value of 0.91. The results of the Spearman-Rank correlation showed however, that lactate and $\mathrm{TCO}_{2}\left(R^{2}=0.6\right)$, lactate and anion gap $\left(R^{2}=0.7\right) ;$ and $\mathrm{TCO}_{2}$ and anion gap $\left(R^{2}=\right.$ $0.7)$ were moderately correlated making interpretation of the role of lactate as an independent predictor difficult. When $\mathrm{TCO}_{2}$ and anion gap were excluded from the model, lactate no longer became a significant predictor $(P=0.2)$. However, when lactate and anion gap are removed from the model and $\mathrm{TCO}_{2}$ added, $\mathrm{TCO}_{2}$ remained a significant variable $(P=0.02)$. Similarly, when anion gap is replaced into the model at the exclusion of lactate and $\mathrm{TCO}_{2}$ it remains a significant variable $(P=$ $0.01)$. Hence the apparent predictor value 


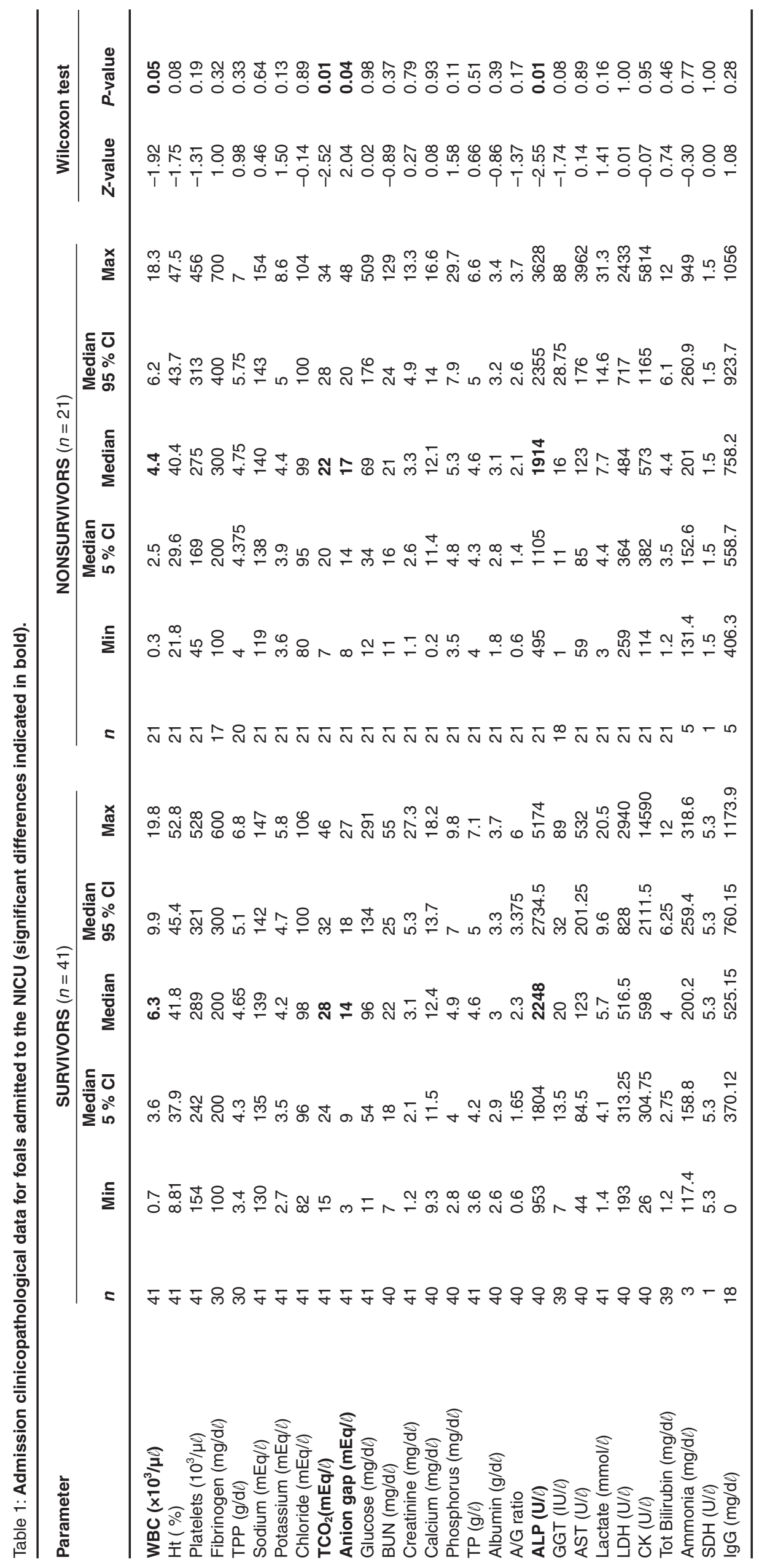

of lactate may be as a result of its association with $\mathrm{TCO}_{2}$ and anion gap rather than its independent effect. Collinearity or multicollinearity can create inaccurate estimates of the regression coefficients, inflate the standard errors of the regression coefficients, deflate the partial $t$-tests for the regression coefficients, give false nonsignificant $P$-values, and degrade the predictability of the model. Because the possibility of collinearity existed between the parameters, the use of individual parameters in the logistic regression model as independent predictors of death need to be done with caution, despite the significant odds ratios. The logistic regression models suggested however that $\mathrm{TCO}_{2}, \mathrm{~A} / \mathrm{G}$ ratio, potassium, sodium $(P<0.05)$; and possibly ALP, WBC count and lactate $(P<0.1)$ were worth examining further as independent predictors of mortality using ROCs. Anion gap, because of its relationship with $\mathrm{TCO}_{2}$ and lactate and the significant difference in median values between foals that died and survived, was also examined further.

The AUROCs for the selected parameters are shown in Table 3. For all parameters the mortality rate (censor) in foals was $34 \%$ and the number of foals included in the calculations was 61 or 62 depending on the parameter. Only anion gap was of any significant value as an individual predictor of foal death. Despite the AUROC for potassium and lactate being in the 60 s, no difference could be shown between their AUROCs and 0.5 at the $95 \%$ confidence level, thus making them inconclusive predictors of death based on this study. Figure 2 shows the AUROCs of anion gap, potassium and lactate relative to 0.5 . Sensitivity indicates the true positive rate (i.e. the probability of correctly predicting a foals death) while 1-specificity indicates the false positive rate (i.e. the probability of incorrectly predicting a foals death). ALP and $\mathrm{TCO}_{2}$ however, appear to be good predictors of survival (AUROC for survival: 70, $P<$ $0.01)$.

Table 4 depicts LOS and costs for foals that survived or died. Foals that survived had longer LOS $(P<0.01)$ and greater median total cost of hospitalisation than nonsurvivors $(P<$ 0.01) while the median cost/day of foals that died was significantly higher than those that survived $(P<$ $0.01)$. There was no difference between median cost/day in foals with 
Table 2: Results of the forward switching logistic regression model for predicting mortality in foals admitted to the NICU.

\begin{tabular}{|c|c|c|c|c|c|c|c|}
\hline Parameter & $\begin{array}{l}\text { Regression } \\
\text { coefficient }(\beta)\end{array}$ & SE & $\begin{array}{l}\text { Wald Z-value } \\
\qquad(\beta=0)\end{array}$ & Wald prob. level & OR & $\begin{array}{c}\text { Lower } 95 \% \\
\mathrm{Cl}\end{array}$ & $\begin{array}{c}\text { Upper } 95 \% \\
\text { Cl }\end{array}$ \\
\hline BO: Intercept & -23.78 & 16.11 & -1.48 & 0.14 & 0.00 & 0.00 & 2441.24 \\
\hline B1: A/G ratio & -1.01 & 0.50 & -2.01 & 0.04 & 0.36 & 0.14 & 0.98 \\
\hline B3: ALP & 0.00 & 0.00 & -1.82 & 0.07 & 1.00 & 1.00 & 1.00 \\
\hline B9: $\mathrm{TCO}_{2}$ & -0.44 & 0.19 & -2.31 & 0.02 & 0.65 & 0.45 & 0.94 \\
\hline B14: Potassium & 1.28 & 0.57 & 2.25 & 0.02 & 3.59 & 1.18 & 10.92 \\
\hline B15: Lactate & -0.32 & 0.18 & -1.78 & 0.08 & 0.73 & 0.51 & 1.03 \\
\hline B16: Sodium & 0.29 & 0.13 & 2.15 & 0.03 & 1.33 & 1.03 & 1.74 \\
\hline B17: WBC & -0.19 & 0.11 & -1.68 & 0.09 & 0.83 & 0.67 & 1.03 \\
\hline B13: Ht & -0.07 & 0.05 & -1.34 & 0.18 & 0.93 & 0.84 & 1.03 \\
\hline
\end{tabular}

$\mathrm{OR}=$ odds ratio $\mathrm{Cl}$ = confidence interval; $\mathrm{SE}=$ standard error; $\mathrm{A} / \mathrm{G}$ ratio = albumin/globulin ratio; $\mathrm{TCO}_{2}=\mathrm{Total}_{\mathrm{CO}}$.

Table 3: AUROCs for selected parameters.

\begin{tabular}{|c|c|c|c|c|c|c|}
\hline Parameter & $\begin{array}{c}\text { Estimate of } \\
\text { empirical AUC }\end{array}$ & SE of AUCs & Lower $95 \% \mathrm{Cl}$ & Upper $95 \%$ Cl & $\begin{array}{l}Z \text {-value to test } \\
\text { AUROC > } 0.5\end{array}$ & $P$-value \\
\hline Anion gap & 0.66 & 0.07 & 0.50 & 0.78 & 2.27 & 0.01 \\
\hline Potassium & 0.62 & 0.07 & 0.45 & 0.74 & 1.60 & 0.05 \\
\hline Lactate & 0.61 & 0.08 & 0.44 & 0.74 & 1.43 & 0.08 \\
\hline Sodium & 0.54 & 0.08 & 0.36 & 0.68 & 0.45 & 0.33 \\
\hline Chloride & 0.49 & 0.08 & 0.32 & 0.63 & -0.15 & 0.44 \\
\hline $\mathrm{A} / \mathrm{G}$ ratio & 0.39 & 0.07 & 0.24 & 0.53 & -2.06 & 0.02 \\
\hline WBC count & 0.35 & 0.08 & 0.19 & 0.49 & -1.98 & 0.02 \\
\hline $\mathrm{TCO}_{2}$ & 0.30 & 0.07 & 0.16 & 0.43 & -2.79 & $<0.01$ \\
\hline ALP & 0.30 & 0.07 & 0.15 & 0.43 & -2.80 & $<0.01$ \\
\hline
\end{tabular}

$\mathrm{Cl}=$ confidence interval; $\mathrm{AUROCs}=$ area under the receiver operate curves; $\mathrm{AUCs}=$ area under the curves; $\mathrm{SE}=\mathrm{standard}$ error $; \mathrm{A} / \mathrm{G}$ ratio $=$ albumin $/ \mathrm{globulin}$ ratio; $\mathrm{TCO}=$ Total $\mathrm{CO}_{2} ; \mathrm{ALP}=$ alkaline phosphatase.

bacteraemia (US\$1133, 95 \% CI: 600-2217) compared to those without (US\$938, $95 \%$ CI: 665-1055) $(P<0.21)$.

\section{DISCUSSION}

On admission of a critically-ill foal to a NICU, the events that follow admission are often a development of the clinical and pathophysiological findings at the time of admission. Clinicians may be placed under great pressure to provide prognostic answers so that clients can make early decisions as to the course of

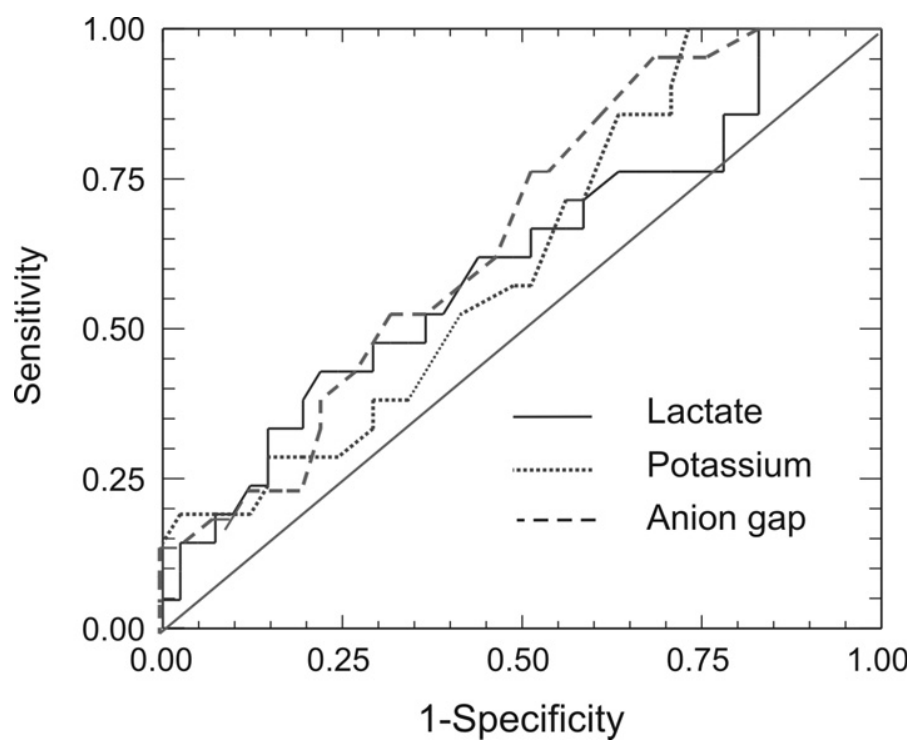

Fig. 2: Receiver operator curves for anion gap, potassium and lactate. actions clinicians should undertake. Understanding the prognostic value of clinicopathological parameters measured at admission is therefore of importance to clinicians so that informed decisions can be made promptly.

Mathematical models have been previously described using selected clinicopathological data to predict outcome in the NICU ${ }^{7,8,11,18}$. Such models have found the following clinicopathological to be significant and indicate a poor prognosis: decreased WBC count ${ }^{8,18}$, increased creati-

nine ${ }^{18}$, increased anion gap ${ }^{11,18}$, decreased neutrophil count ${ }^{7,8}$, decreased venous $\mathrm{PO}_{2}{ }^{11}$, decreased albumin ${ }^{8}$, decreased glu$\operatorname{cose}^{8}$, decreased blood $\mathrm{pH}^{8}$ and negative base excess ${ }^{8}$. In this study, WBC counts were higher in surviving foals as compared with dead foals (mean: $6.3 \times 10^{3} v s$ $4.4 \times 10^{3}$ cells $\left./ \mu l, P=0.05\right)$. Previous studies have also reported higher WBC counts in survivors vs nonsurvivors: $7.4 \times 10^{3} v s$ $3.3 \times 10^{3}$ cells $/ \mu \ell[P<0.05]^{18}, 9.976 \times 10^{3} v s$ $6.791 \times 10^{3} \mathrm{cells} / \mu l[P=0.02]^{7}, 11.1 \times 10^{3} v \mathrm{~s}$ $10.5 \times 10^{3}$ cells $/ \mu l[P=0.42]^{11}, 10 \times 10^{3} v s$ $6.55 \times 10^{3}$ cells $/ \mu l[P=0.085]^{8}$.

Septicaemia, endotoxaemia and prematurity may cause leukopenia ${ }^{12,15}$. Anion gap has been previously used to predict survival of foals admitted to a NICU ${ }^{11,18}$, foals with radiographic evidence of pulmonary disease ${ }^{2}$ and adult horses with colic ${ }^{3}$. Lower anion gap has been reported in survivors vs nonsurvivors (mean: 15.8 vs $22.4 \mathrm{mEq} / \mathrm{l}, P=0.002)^{11}$, while an anion gap $\geq 20 \mathrm{mEq} / \ell(P<0.001)$ was significantly associated with nonsurvival ${ }^{2}$. These findings are consistent with the present study where survivors had lower anion gap compared with nonsurvivors (mean: 14 vs $17 \mathrm{mEq} / \ell[P<$ $0.04]$ ). In foals, a high anion gap is an indicator of metabolic acidosis ${ }^{16}$ due to hypoxia and reduced tissue perfusion. Bicarbonate is the major component of $\mathrm{TCO}_{2}$ and changes in $\mathrm{TCO}_{2}$ are 


\begin{tabular}{|c|c|c|c|c|c|c|c|}
\hline & \multicolumn{3}{|c|}{ Survivors } & \multicolumn{3}{|c|}{ Nonsurvivors } & \multirow[t]{2}{*}{$P$-value } \\
\hline & Lower 95 \% Cl & Median & Upper $95 \%$ Cl & Lower $95 \%$ Cl & Median & Upper 95 \% Cl & \\
\hline Median days in hospital & 4 & 5 & 6 & 1 & 1 & 1 & $<0.01$ \\
\hline Median total cost (US\$) & 2370 & 3038 & 4058 & 1457 & 1793 & 2217 & $<0.01$ \\
\hline Median cost/d (US\$) & 607 & 671 & 876 & 1220 & 1505 & 1822 & $<0.01$ \\
\hline
\end{tabular}

$\mathrm{Cl}=$ confidence interval.

interpreted as changes in bicarbonate concentration'. In this report, $\mathrm{TCO}_{2}$ was higher in survivors vs nonsurvivors (mean: 28 vs $22 \mathrm{mEq} / l, P=0.01$ ), similar to a previous report ${ }^{8}$ (mean: 30.5 vs $26 \mathrm{mEq} / \mathrm{l}$, $P=0.5)$. ALP was also found to be higher in survivors vs nonsurvivors (2248 vs 1914 $\mathrm{mmol} / \ell, P=0.01)$ as reported previously ${ }^{11}$. Although ALP has a broad specificity with isoenzymes present in liver, bone, intestine, kidney and placenta, the observed increase may be due to ingestion of colostrum ${ }^{6}$. Other causes for increased ALP include cholestasis, corticosteroids, nonsteroidal drugs, bone disease and neoplasia ${ }^{6}$.

Overall survival rates have been reported as $83 \%(n=577)^{18}, 68 \%(n=99)^{7}, 66 \%$ $(n=56)^{11}, 45 \%(n=65)^{8}$ and $36 \%(n=$ $160)^{21}$ compared to $66 \%(n=62)$ in this study. An increased mortality rate was recorded in the NICU on day 2 post admission. Often, NICUs may experience the highest mortality rates in the first 24 hours following admission due to the advanced disease process or failure to respond to treatment. It was surprising that a second increase in mortality rate was reported on day 6 in this study as the final days of hospitalisation are essentially recuperative and may have been caused by significant organ damage sustained prior to admission or in the first 24 hours after admission that caused continued microcellular and vascular damage.

The total cost of treating a foal that survived was higher than nonsurvivors. However, this is probably a function of the survival time as foals that survived had longer LOS. The total cost of hospitalisation of foals that died was significantly higher than those that survived and may be because the admission phase of care involves more expensive diagnostics and more intervention due to the severity of the disease process. Interestingly, the presence of bacteraemia did not influence cost in this study.

Several limitations are recognised in this study. Due to the retrospective nature, the study may have suffered from a selection bias, while interpretation of single measurements may have had limitations and thus serial measurements may have had greater prognostic value. A specific population of foals was sampled from 1 region admitted only to a single NICU. In addition, a confounding effect of small sample volume with similar disease aetiology may have affected the results.

This study demonstrated the clinical usefulness of measuring clinicopathological data (WBC count, $\mathrm{TCO}_{2}, \mathrm{ALP}$ and anion gap) in neonates admitted to an equine NICU before initiation of treatment and that anion gap was an independent predictor of neonatal mortality. As mortality was higher in foals following forced extraction during resolution of dystocia, the veterinary internist should consider such foals 'high risk'. Also, the analysis of patient costs confirmed and quantified the higher NICU daily cost for nonsurvivors.

\section{ACKNOWLEDGEMENTS}

The authors wish to acknowledge the dedication of the internists, interns, fellows and nursing support staff of the McGee Medical Center who provided the best possible care for the neonates in the NICU. A portion of this work was presented as an abstract at the 2006 International Veterinary Emergency and Critical Care Symposium in San Antonio, Texas, USA.

\section{REFERENCES}

1. Anderson T W, Darling D A 1954 A test of goodness-of-fit. Journal of the American Statistical Association 49: 765-769

2. Bedenice D, Heuwieser W, Solano M, Rand W, Paradis M R 2003 Risk factors and prognostic variables for survival of foals with radiographic evidence of pulmonary disease. Journal of Veterinary Internal Medicine 17: 868-875

3. Bristol D G 1982 The anion gap as a prognostic indicator in horses with abdominal pain. Journal of the American Veterinary Medical Association 181: 63-65

4. Corley K T T, Donaldson L L, Furr M O 2005 Arterial lactate concentration, hospital survival, sepsis and SIRS in critically ill neonatal foals. Equine Veterinary Journal 37: 53-59

5. DeLong E R, DeLong D M, Clarke-Pearson D L 1988 Comparing the areas under two or more correlated receiver operating characteristic curves: a nonparametric approach. Biometrics 44: 837-845

6. Duncan J R, Prasse K W, Mahaffey E A (eds) 1994 Veterinary laboratory medicine: Clinical pathology (3rd edn). Iowa State University Press, Ames: 130-151

7. Furr M, Tinker M K, Edens L 1997 Prognosis for neonatal foals in an intensive care unit.
Journal of Veterinary Internal Medicine 11: 183-188

8. Gayle J M, Cohen N D, Chaffin M K 1998 Factors associated with survival in septicemic foals: 65 cases (1988-1995). Journal of Veterinary Internal Medicine 12: 140-146

9. George J W 1994 Water, electrolytes and acid base. In Duncan J R, Prasse K W, Mahaffey E A (eds) Veterinary laboratory medicine: clinical pathology (3rd ed). Iowa State University Press, Ames: 94-111

10. Gibbons J 1976. Nonparametric methods for quantitative analysis. Holt, Rinehart and Winston. New York

11. Hoffman A M, Staempfli H R, Willan A 1992 Prognostic variables for survival of neonatal foals under intensive care. Journal of Veterinary Internal Medicine 6: 89-95

12. Jeffcot L B, Rossdale P D, Leadon D P 1982 Haematological changes in the neonatal period of normal and induced premature foals. Journal of Reproduction and Fertility Supplement 32: 537-544

13. Klein J P, Moeschberger M L (eds) 1997 Survival analysis. Springer-Verlag, New York

14. Koliski A, Cat I, Giraldi D J, Cat M L 2005 Blood lactate concentration as prognostic marker in critically ill children. Jornal de Pediatria 81: 287-292

15. Koterba A M, Brewer B D, Tarplee F A 1984 Clinical and clinicopathological characteristics of the septicemic neonatal foal: review of 38 cases. Equine Veterinary Journal 16: 376-382

16. Meyer D J, Harvey J W (eds) 1998 Veterinary laboratory medicine: interpretation and diagnosis (2nd edn). W B Saunders, Philadelphia

17. Rocktaeschel J, Morimatsu H, Uchino S, Bellomo R 2004 Unmeasured anions in critically ill patients: Can they predict mortality? Critical Care Medicine 31: 2131-2136

18. Rohrbach B W, Buchanan B R, Drake J M, Andrews F M, Bain F T, Byars D T, Bernard W V, Furr M O, Paradis M R, Lawler J, Giguere S, Dunkel B 2006 Use of a multivariate model to estimate the probability of discharge in hospitalized foals that are 7 days of age or less. Journal of the American Veterinary Medical Association 228: 17481756

19. Shapiro S S, Wilk M B 1965 An analysis of Variance test for normality. Biometrika 52: 591-611

20. Steel C M, Hunt A R, Adams P L, Robertson I D, Chicken C, Yovich J V, Stick J A 1999 Factors associated with prognosis for survival and athletic use in foals with septic arthritis: 93 cases (1987-1994). Journal of the American Veterinary Medical Association 215: 973-977

21. Welschen S E, de Bruijn C M, van Oldruitenborgh-Oosterbaan M M 2005 The short- and long-term results of the intensive care of 160 sick neonatal foals. Tijdschrift voor diergeneeskunde 130: 168-173

22. Zhou X, Obuchowski N, McClish D 2002 Statistical methods in diagnostic medicine. John Wiley \& Sons, New York 